ISSN 1112-9867

http://www.jfas.info

\title{
ANTIOXIDANT ACTIVITY OF HIGH-DENSITY LIPOPROTEIN (HDL) USING DIFFERENT IN VITRO ASSAYS
}

W. N. W. Yusoff ${ }^{1}$, N. A. A. Bakar', S. Muid ${ }^{1}$, A. M. Ali ${ }^{3}$, G. R. A. Froemming ${ }^{1,2}$ and H. Nawawi $i^{1,2, *}$

${ }^{1}$ Institute for Pathology, Laboratory and Forensic Medicine (I-PPerForM), Universiti Teknologi MARA, 47000 Sungai Buloh, Selangor, Malaysia ${ }^{2}$ Faculty of Medicine, UniversitiTeknologi MARA, 47000 Sungai Buloh, Selangor, Malaysia ${ }^{3}$ Faculty of Bioresources and Food Industry, Universiti Sultan ZainalAbidin (UniSZA), 20400 Terengganu, Malaysia

Published online: 10 November 2017

\begin{abstract}
The role of HDL in reverse cholesterol transport is well known but other atheroprotective mechanism, e.g. antioxidant capacities of HDL is not clear. The aim of this study is to determine the effects of different doses of HDL on antioxidant activities assay. HDL was isolated from plasma by single step-ultracentrifugation. The antioxidant activity of different doses of HDL were measured by ferric thiocyanate (FTC), 2,2-diphenyl-1-picrylhydrazyl (DPPH) and dichlorofluorescein (DCF) tests. Coincubation of HDL with LDL showed longer lag time and lower reaction rate in a dose-dependent manner compared to LDL alone $(\mathrm{p}<0.05)$. HDL had inhibitory effects on radical oxygen species (ROS) production but did not exhibit free radical scavenging activities. HDL is a potent antioxidant in terms of inhibition of lipid peroxidation, ROS production and LDL oxidation. These may to some extent add to the antiatherogenic beyond reverse-cholesterol transport properties of HDL.
\end{abstract}

Author Correspondence, e-mail: hapizah.nawawi@mail.com doi: http://dx.doi.org/10.4314/jfas.v9i6s.24 
Keywords: high-density lipoprotein; reverse cholesterol transport; apolipoprotein A1; antioxidant; in vitro.

\section{INTRODUCTION}

Lipids are circulated in the human body by an intricate lipid transport framework containing five fundamental classes of lipoproteins which from the smallest in molecular size to biggest are high-density lipoproteins (HDL), low density lipoproteins (LDL), intermediate density lipoproteins, very low density lipoproteins and chylomicrons [1]. HDL plays a vital part against the progression of atherosclerosis and cardiovascular illnesses and high plasma levels of HDL are inversely connected with the occurrence of these pathologies [2-3]. In Fig. 1, the structure of the HDL is depicted [4].

Present reviews have demonstrated that few lipid peroxidation products related with oxidized LDL (ox-LDL) display an assortment of biological activities possibly embroiled in atherogenesis. HDL applies their anti-atherogenic, anti-inflammatory and cardioprotective activities through numerous mechanisms [5-7]. Besides, to invert cholesterol transport which decreases tissue cholesterol level, HDL can contravene the atherogenic effect of ox-LDL by controlling membrane cholesterol content, restraining low density lipoprotein (LDL) oxidation and bringing down inflammatory response [8].

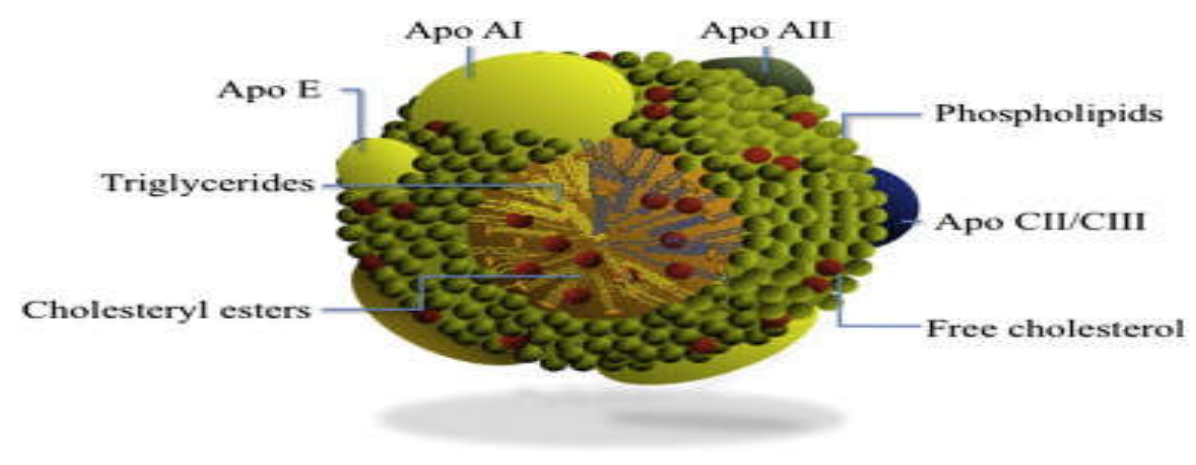

Fig.1. The structure of HDL

Other than that, HDL can also counteract endothelial cell dysfunction and activation, keep up the respectability of the endothelial cell lining, and diminish the deleterious impact of ox-LDL and the levels of thrombogenic factors [9-10]. The impacts of HDL could result from HDL-related particles or from their own properties of cell signaling modulator or lipid transporter. 
All described properties of HDL have been identified in cell culture, animal models, human studies and even in patient populations [8]. It is generally believed that a large number of the beneficial impacts of HDL may be related to their part in reverse cholesterol transport, however little consideration has been paid to its antioxidant property. Therefore, the aim of this study was to analyze the antioxidant properties of HDL. In addition, the underlying mechanisms of its antioxidant properties were to be resolved using distinctive assays-ferric thiocyanate (FTC) assay, 2,2-diphenyl-1-picrylhydrazyl (DPPH) free radical scavenging activity assay as well as the 2', 7'- dichlorofluorescindiacetete (DCFHDA) assay. Tocotrienol (TCT), a member of the vitamin E family, butylatedhydroxytoluene (BHT) and Vitamin C-all are known effective antioxidants-were used as positive controls. Possible interactions between HDL proteins in inhibiting LDL oxidation are determined by coincubations of HDL and LDL subjected to copper sulphate.

\section{METHODOLOGY}

\subsection{Materials}

Potassium bromide $(\mathrm{KBr})$, sodium chloride $(\mathrm{NaCl})$, copper (II) sulphatepentahydrate $\left(\mathrm{CuSO}_{4} .5 \mathrm{H}_{2} \mathrm{O}\right)$, ethylenediaminetetraacetic acid (EDTA), linoleic acid, ammonium thiocyanate, butylatedhydroxytoluene (BHT), Vitamin C, DPPH, DCFHDA, Hanks' balanced salt solution without phenol red and Escherichia coli LPS from serotype 055:B5 were purchased from Sigma-Aldrich, USA. TCT was supplied by Sime Darby Sdn. Bhd, Malaysia. Ethanol was purchased from Scharlau, Barcelona, Spain. Ferrous chloride and 3.5\% hydrochloric acid were obtained from Merck, USA. Methanol was acquired from Merck, Germany. RAW 264.7 cells were purchased from American Type Culture Collection (ATCC), USA. Dulbecco's Modified Eagle Media (DMEM) containing $4 \mathrm{mM}$ L-glutamine, $4.5 \mathrm{~g} / \mathrm{L}$ glucose and $1.5 \mathrm{~g} / \mathrm{L}$ sodium bicarbonate were obtained from Flow Lab, Australia. Recombinant mouse interferon Gamma was acquired from BD Pharmingen, USA. 3-(4,5-dimethylthiazol-2-Y1)-2,5-diphenyltetrazolium bromide (MTT) was bought from Sigma-Aldrich, USA.

\subsection{HDL Isolation}

Blood from healthy donors were collected by venipuncture. Plasma was separated from blood 
by centrifugation at $3,000 \mathrm{rpm}$ at $4{ }^{\circ} \mathrm{C}$ for 10 minutes and pooled. The pooled plasma was fixed to a density of $1.21 \mathrm{~g} / \mathrm{ml}$ by integrating $1.1 \mathrm{ml}$ plasma to $0.3270 \mathrm{~g}$ of $\mathrm{KBr}$ and mixed tenderly by vortexing. An irregular density gradient was made by overlaying the plasma solution $(1.1 \mathrm{ml})$ with $2.2 \mathrm{ml}$ of buffer $(\mathrm{NaCl} 0.15 \mathrm{M}$, EDTA $1.0 \%, \mathrm{pH} 7.4)$ in a $3.3 \mathrm{ml}$ ultracentrifuge tube $(\mathrm{d}=1.006 \mathrm{~g} / \mathrm{ml})$. The ultracentrifuge tube was sealed and centrifuged at $100,000 \mathrm{rpm}$ at $4^{\circ} \mathrm{C}$ for 40 minutes in a Beckman Optima TLX centrifuge (Beckman Coulter, Palo Alto, California). After centrifugation, the tube was carefully removed from the rotor and put in a vertical position. The yellow-orange HDL fraction remained at the base of the tube. The HDL fraction was collected using a $25 \mathrm{G}$ needle and $1 \mathrm{ml}$ syringe and placed into a test tube on ice. Afterwards, the HDL fraction was placed into a nitro cellulose bag and dialyzed in a glass bottle filled with phosphate buffer at $4^{\circ} \mathrm{C}$ for 24 hours. The total protein concentration was determined using the automated chemistry analyzer (Cobas Integra 400, Roche Diagnostics, Switzerland).

\subsection{FTC Assay}

Four $\mathrm{ml}$ of various concentrations of HDL $(20-120 \mathrm{mg} / \mathrm{dl}), 8 \mathrm{ml}$ of $0.05 \mathrm{M}$ of phosphate buffer with $\mathrm{pH} 7.0,4.1 \mathrm{ml}$ of $2.52 \%$ linoleic acid dissolved in absolute ethanol and $3.9 \mathrm{ml}$ of deionized water were mixed in a screw cap vial. The vial was heated at $40^{\circ} \mathrm{C}$ in the dark. Then, $0.1 \mathrm{ml}$ of this solution was added to a reaction mixture which contained $9.7 \mathrm{ml}$ of $75 \%$ ethanol, $0.02 \mathrm{M}$ ferrous chloride in $3.5 \%$ hydrochloric acid and $0.1 \mathrm{ml}$ of $30 \%$ ammonium thiocyanate. The absorbance was measured at $500 \mathrm{~nm}$ for every 24 hours until maximum absorbance of the control is reached. The solvent was used as negative control. Tocotrienol and BHT were used as positive controls.

\subsection{DPPH Free Radical Scavenging Activity Assay}

DPPH solution was prepared by dissolving $5 \mathrm{mg}$ of DPPH in $2 \mathrm{ml}$ of methanol and kept in the dark at $4^{\circ} \mathrm{C}$. A concentration of $1000 \mu \mathrm{g} / \mathrm{ml}$ of the HDL stock solution was prepared by diluting the HDL extract in methanol. The stock solution of HDL $(10000 \mu \mathrm{g} / \mathrm{ml})$ was incorporated into the well and it was further diluted in a 96-well microplate. Stock solutions of BHT and Vitamin C at the concentration of $1000 \mu \mathrm{g} / \mathrm{ml}$ were used as positive controls. Five microlitre of DPPH in methanol was incorporated into every well and left it for 30 minutes in the dark. The absorbance was measured using ELISA reader (EL340 Biokinetic reader, 
Bio-Tek Instrumentation) at $517 \mathrm{~nm}$. Percentage inhibition was calculated by using the following formula:

Percentage Inhibition $=\underline{\text { Optical Density }(O D)(D P P H)}-$ OD $(D P P H+$ sample $) \times 100 \%$

$$
\text { OD (DPPH) }
$$

The antioxidant activity of HDL was expressed in terms of $\mathrm{IC}_{50}$ required to inhibit DPPH radical formation by $50 \%$ [10a-b].

\subsection{DCFHDA Assay}

Cells were grown in DMEM containing $10 \%$ FBS and $1 \%$ penicillin/streptomycin at $37^{\circ} \mathrm{C}$ in a humidified $\mathrm{CO}_{2}$ incubator. The RAW 264.7 cells were plated at $0.5 \times 106 / \mathrm{ml}$ in a 96-well microplate and incubated for 24 hours to allow cell adherence. To determine that the observed DCFHDA inhibition was not an erroneous positive because of cytotoxic effects, an earlier cytotoxicity assay was performed. Cell viability was assessed by MTT assay. Intracellular oxidative stress was monitored by the DCFHDA method. RAW 264.7 cells were incubated with various concentrations of HDL (10-120 mg/dl), stimulated with LPS and IFN- $\gamma$ in a humidified $\mathrm{CO}_{2}$ incubator at $37^{\circ} \mathrm{C}$ for 18 hours in a black 96-well microplate. After that, each well was loaded with $100 \mu \mathrm{l}$ of $100 \mu \mathrm{M}$ DCFHDA. The intracellular fluorescence intensity was observed for at $485 \mathrm{~nm}$ excitation and $530 \mathrm{~nm}$ emission filters for 30 minutes. This assay measured the ability of different concentrations of HDL to inhibit the production of intracellular $\mathrm{H}_{2} \mathrm{O}_{2}$ as reflected by the reduction in the production of fluorescence intensity by dichlorofluorescein (DCF).

\subsection{Oxidation of LDL}

The isolated LDL was adjusted to $50 \mu \mathrm{g}$ LDL protein/ml by PBS and incubated with different concentrations of HDL (20, 40, 60 and $80 \mu \mathrm{g} / \mathrm{ml})$. The HDL and LDL mixture were oxidized with $5.0 \mu \mathrm{M} \mathrm{CuSO}_{4} .5 \mathrm{H} 2 \mathrm{O}$. Absorbance increment was measured at $234 \mathrm{~nm}$ by a spectrophotometer. Lag phase expressed in minutes is the interval between the addition of $\mathrm{CuSO}_{4}$ and the commencement of the extensive oxidation and was quantified on the base of the intercept between the baseline and the tangent to the fast oxidation phase. The proliferation rate [expressed in changes in absorbance $(\mathrm{Abs} / \mathrm{min})$ ] is the maximal rate of LDL oxidation detected in the kinetic curve. 


\subsection{Statistical Analysis}

Data were reported as mean \pm SD and analyzed using the SPSS statistical package version 24 .

The effects of various concentrations of HDL on antioxidant assay were tested with analysis of variance (ANOVA). Two different groups comparison was analyzed by independent t-test. All levels of significance were set at $\mathrm{p}<0.05$.

\section{RESULTS AND DISCUSSION}

The ability of HDL to inhibit lipid peroxidation was tested using the FTC method. The results of the experiments are shown in Fig. 1 illustrated the absorbance values of different concentrations of HDL. HDL ranging from 20-120 mg/dl were used as positive controls and solvents without HDL, TCT or BHT were used as the negative controls. The lower absorbance value of test sample indicates higher antioxidant activity. All HDL concentration tested showed significant antioxidant capabilities. HDL at lower concentrations $(20-60 \mathrm{mg} / \mathrm{dl})$ resulted in a significant inhibition of peroxide generation but at higher concentrations (80-120 $\mathrm{mg} / \mathrm{dl}$ ) resulted in significant absorbance reduction to a greater extent than that for HDL at lower concentrations $(20-60 \mathrm{mg} / \mathrm{dl})(\mathrm{p}<0.05$ versus $\mathrm{p}<0.001$ compared to negative controls respectively) (Fig. 1). Similarly, HDL at higher concentrations $(80-120 \mathrm{mg} / \mathrm{dl})$ showed greater percentage of inhibition of lipid peroxidation than that for HDL at lower concentrations. TCT and BHT as the positive controls appeared to scavenge peroxides and avert lipid peroxidation as demonstrated by the low absorbance $(0.15 \pm 0.008$ and $0.08 \pm 0.001$ respectively). These were reflected by the non-significant differences in lipid peroxidation percentage inhibition between higher HDL concentrations and the positive controls, but significantly lower percentage inhibition in lower HDL concentration compared to positive controls (Fig. 2). Fig. 2 showed the percentage inhibition of various HDL concentrations as compared with the negative control. The addition of TCT and BHT to the reaction resulted in an inhibition of peroxides formation by $89.6 \pm 1.7 \%$ and $94.4 \pm 0.1 \%$ respectively. HDL at a concentration of 80 $\mathrm{mg} / \mathrm{dl}$ showed the highest percentage inhibition with $71.0 \pm 1.2 \%$. 


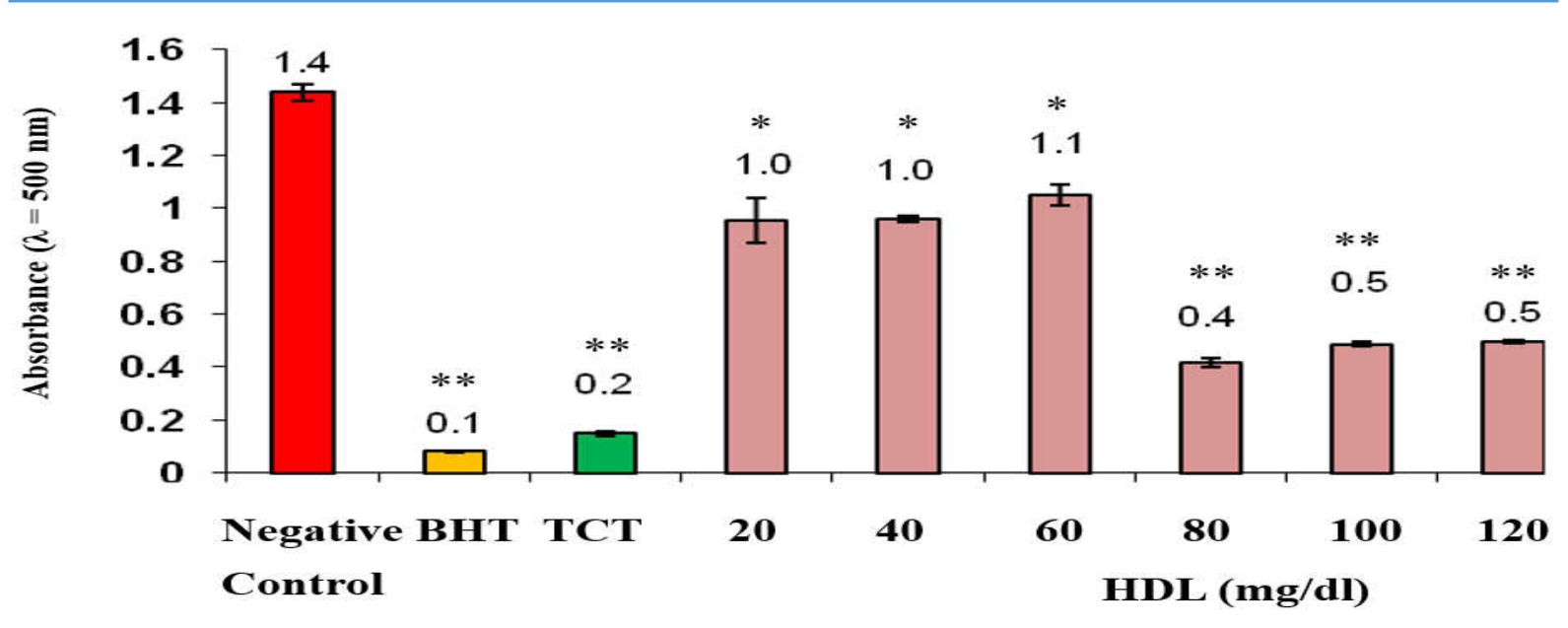

Fig.1. Absorbance values of different concentrations of HDL (20-120 mg/dl) as measured by FTC method

Each set of experiment was performed in triplicates. BHT and TCT were used as positive controls. Results are presented as mean \pm SD compared to negative control, ${ }^{*} \mathrm{p}<0.05,{ }^{* *} \mathrm{p}<0.001$. (HDL: High-density lipoprotein; BHT: Butylatedhydroxytoluene; TCT: Tocotrienol)

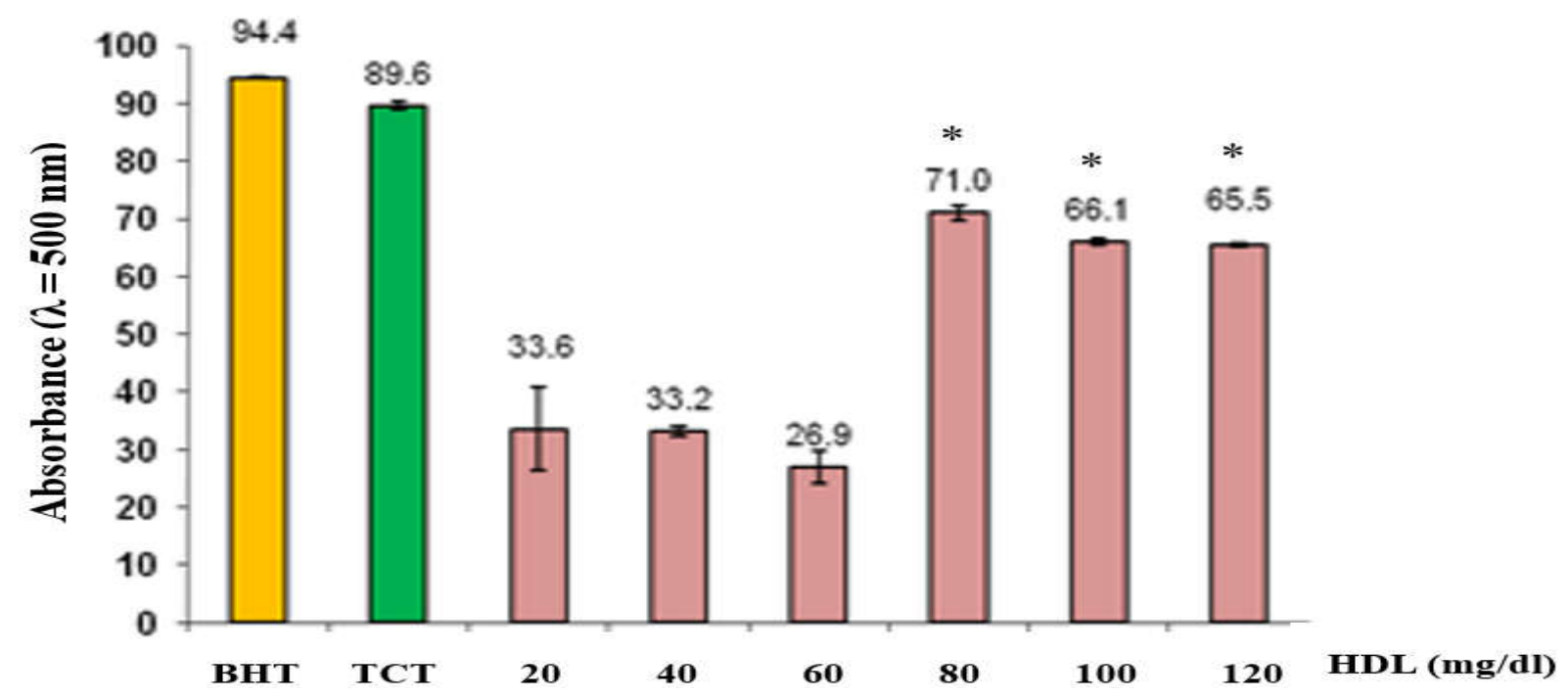

Fig.2. HDL induced inhibition of lipid peroxidation. Percentage inhibition was expressed as percentage of negative control. BHT and TCT were used as positive controls. Data are expressed as mean \pm SD. Across HDL concentrations, there were significant antioxidant activity. ${ }^{*}$ p $<0.05$ compared to BHT and TCT (HDL: High-density lipoprotein; BHT:

Butylatedhydroxytoluene; TCT: Tocotrienol)

The DPPH radical was broadly utilized as part of the model framework to examine the scavenging activities of a few natural compounds, for example, phenolics and anthocyanins. DPPH radical is scavenged by antioxidants through the donation of a proton composing the decreased DPPH. The color changes from purple to yellow after reduction, which can be 
measured by its decrease of absorbance at wavelength $517 \mathrm{~nm}$. Radical-scavenging action increases with incrementing percentage of free radical inhibition. The outcomes for HDL radical scavenging capacity is represented in Fig. 3, demonstrating the dosage reaction curve for the radical-scavenging activity of HDL, TCT, vitamin $\mathrm{C}$ and BHT utilizing the DPPH colorimetric technique. It was found that radical-scavenging activity of HDL diminished as the HDL concentration increased (Fig. 4). The highest radical-scavenging activity was TCT (86.2 $\pm 0.7 \%)$ followed by vitamin C $(85.8 \pm 0.4 \%)$, BHT $(82.7 \pm 2.0 \%)$ and HDL $(6.2 \pm 0.4 \%)$. Percentage inhibition of DPPH radical by HDL significantly differ $(\mathrm{p}<0.05)$ compared to all the controls (Fig. 5). The $\mathrm{IC}_{50}$ for HDL cannot be determined as the maximum percentage inhibition was $<50 \%$. In the same assay, positive controls consisting of TCT, BHT and vitamin $\mathrm{C}$ shown potent antioxidant activity with $\mathrm{IC}_{50}$ of $23.7,23.1$ and $13.7 \mu \mathrm{g} / \mathrm{ml}$ respectively (Fig. 6). In the DPPH assay, it was demonstrated that the radical-scavenging action of HDL diminished as the HDL concentration increased further.

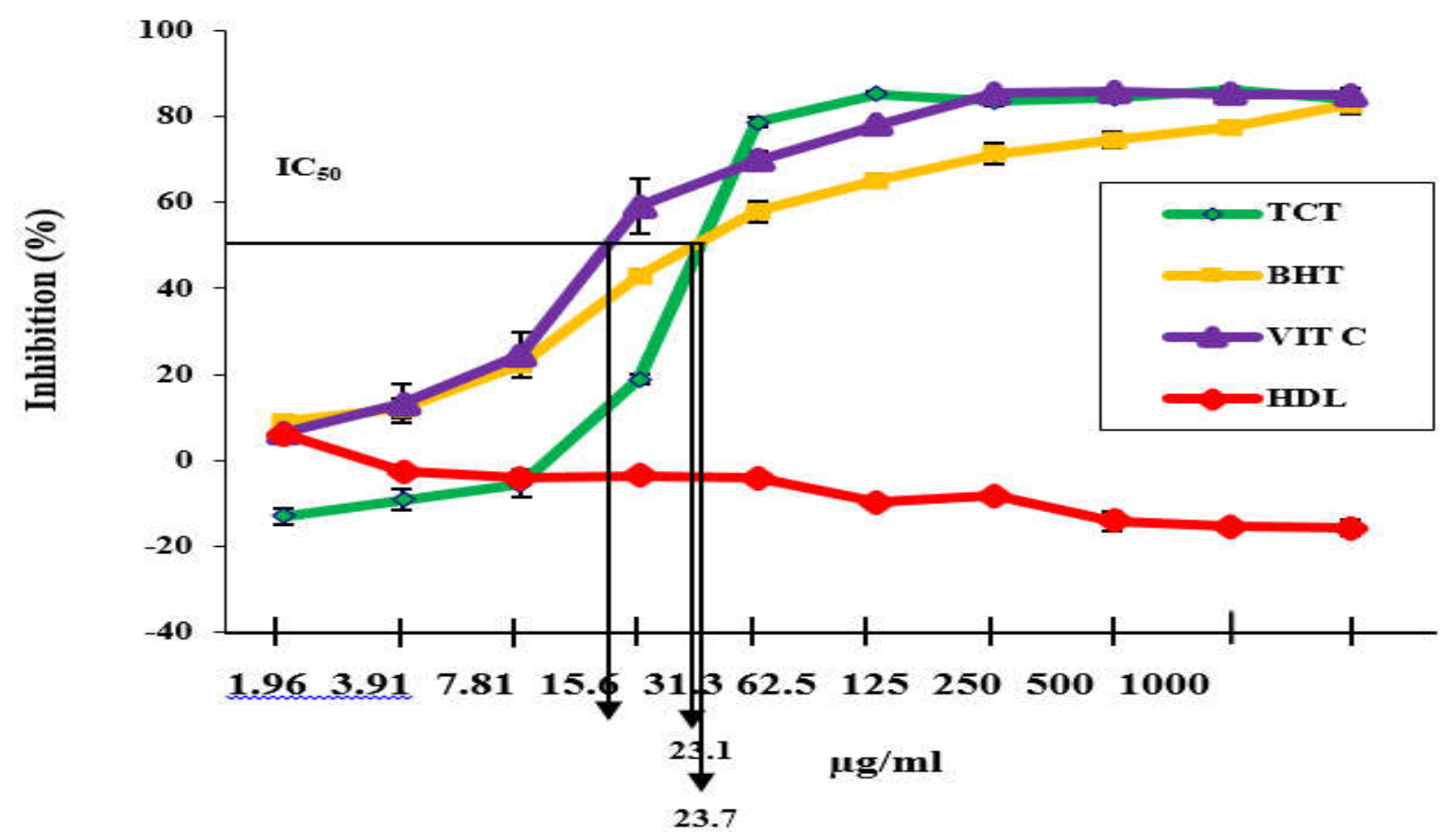

Fig.3. Percentage inhibition of different concentration of HDL, TCT, BHT and vitamin C in DPPH free radical scavenging assay. The graphs also showed $\mathrm{IC}_{50}$ of TCT, BHT and vitamin

$\mathrm{C}$ however $\mathrm{IC}_{50}$ for HDL cannot be determined as the percentage inhibition of HDL is $<50 \%$.(HDL: High-density lipoprotein; BHT: Butylatedhydroxytoluene; TCT: Tocotrienol) 


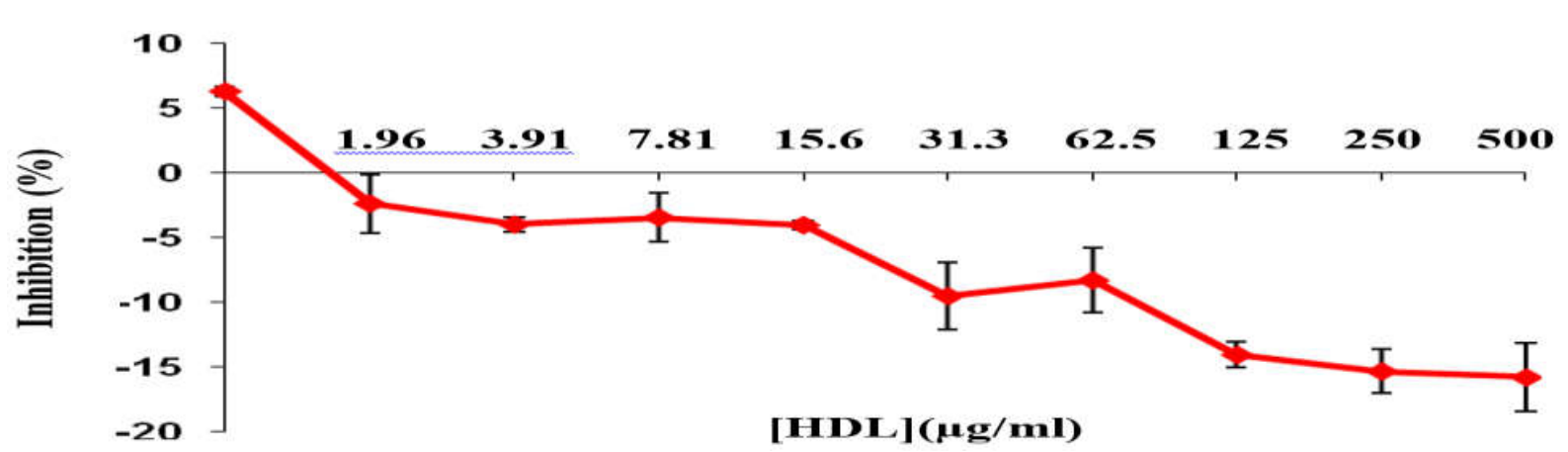

Fig.4. Percentage inhibition of different concentrations of HDL in DPPH free radical scavenging assay. The graph shows that the free radical-scavenging activity of HDL decreased with increasing HDL. $\mathrm{IC}_{50}$ for HDL cannot be determined as the maximum percentage

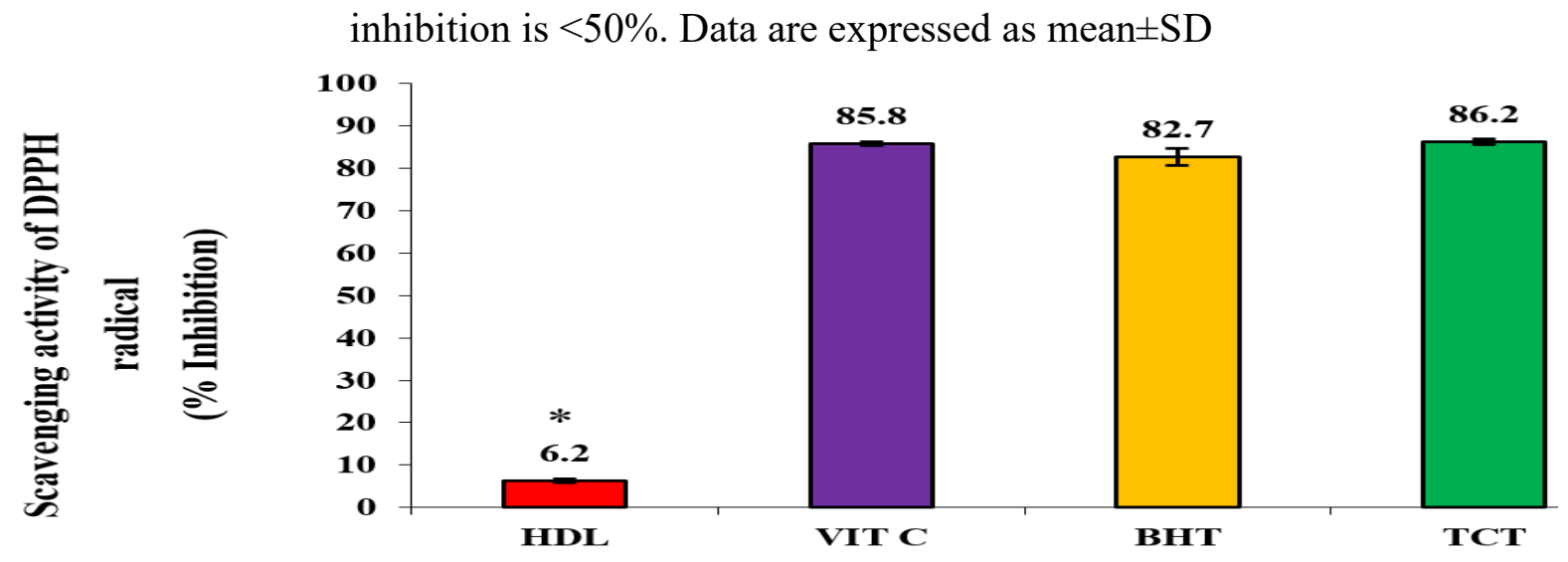

Fig.5. The highest percentage scavenging activity of HDL, vitamin C, BHT and TCT against DPPH radical. Percentage inhibition of DPPH radical by HDL was significantly lower $\left({ }^{*} \mathrm{p}<0.05\right)$ compared to all positive controls. Data are expressed as mean $\pm \mathrm{SD}(\mathrm{HDL}$ : High-density lipoprotein; BHT: Butylatedhydroxytoluene; TCT: Tocotrienol)

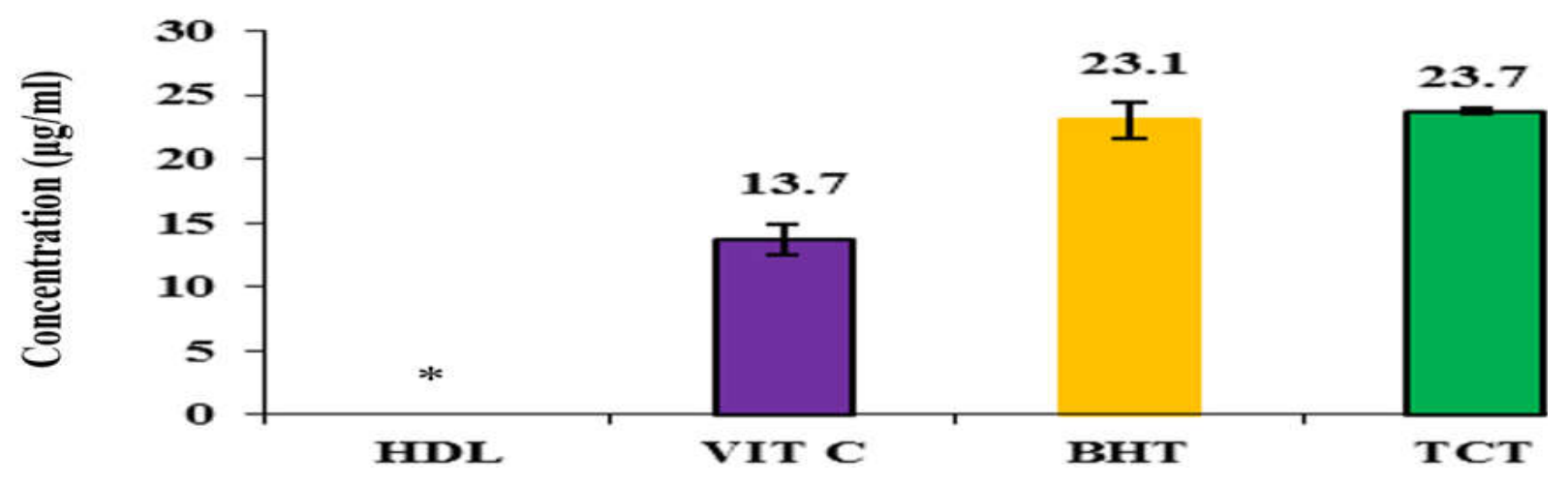

Fig.6. The comparison of $\mathrm{IC}_{50}$ of $\mathrm{HDL}$ with vitamin $\mathrm{C}$, BHT and TCT in DPPH assay. $\mathrm{IC}_{50}$ for HDL cannot be determined as the percentage inhibition of HDL is $<50 \%$. Results are reported as mean \pm SD compared to vitamin $\mathrm{C}$, BHT and TCT, ${ }^{*} \mathrm{p}<0.05$ (HDL: High-density lipoprotein; BHT: Butylatedhydroxytoluene; TCT: Tocotrienol) 
The RAW 264.7 cell cytotoxicity was performed with various concentrations of HDL. The results demonstrated that cell viability was impervious to HDL concentration (Fig. 7). HDL concentrations of $10-120 \mathrm{mg} / \mathrm{dl}$ have more than $90 \%$ of cell viability. The DCFHDA assay showed the ability of the HDL (10-120 mg/dl) to inhibit production of DCF in RAW 264.7 cell line after stimulation with lipopolysaccharide (LPS) and interferon-gamma (IFN- $\gamma$ ) Fig. 8. The lower percentage of DCF fluorescence indicates lower radical oxygen species (ROS) production. The lowest percentage of DCF fluorescence generation was at $100 \mathrm{mg} / \mathrm{dl} \mathrm{HDL}$ concentration compared to negative control $(13.3 \pm 0.3 \%$ versus $19.3 \pm 1.4 \%, \mathrm{p}<0.05)$. HDL at $100 \mathrm{mg} / \mathrm{dl}$ has been shown to inhibit the production of hydrogen peroxide $\left(\mathrm{H}_{2} \mathrm{O}_{2}\right)$ with percentage inhibition of $49.3 \pm 1.7 \%$ (Fig. 9). BHT as a positive control expressed the lowest percentage of DCF fluorescence production $(7.7 \pm 1.4 \%)$ and showed the highest percentage inhibition of $70.8 \pm 1.4 \%$.

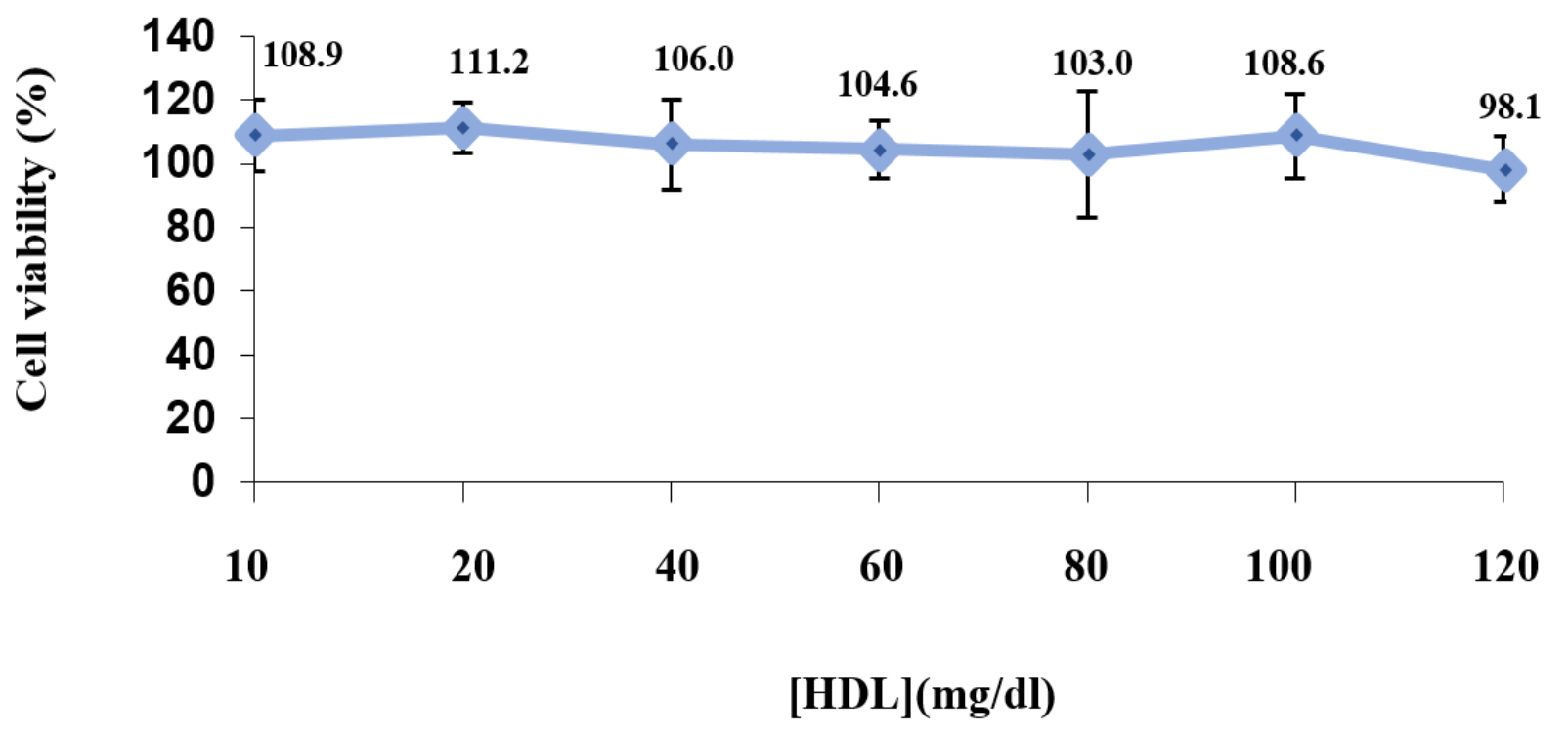

Fig.7.The cell viability of RAW 264.7 when treated with different concentrations of HDL (10-120 mg/dl). The absorbance of the control cells (RAW 264.7 with the absence of HDL) was considered as being 100\% cell viability. Throughout all HDL concentrations, cell viability was more than $90 \%$. Data are expressed as mean \pm SD 


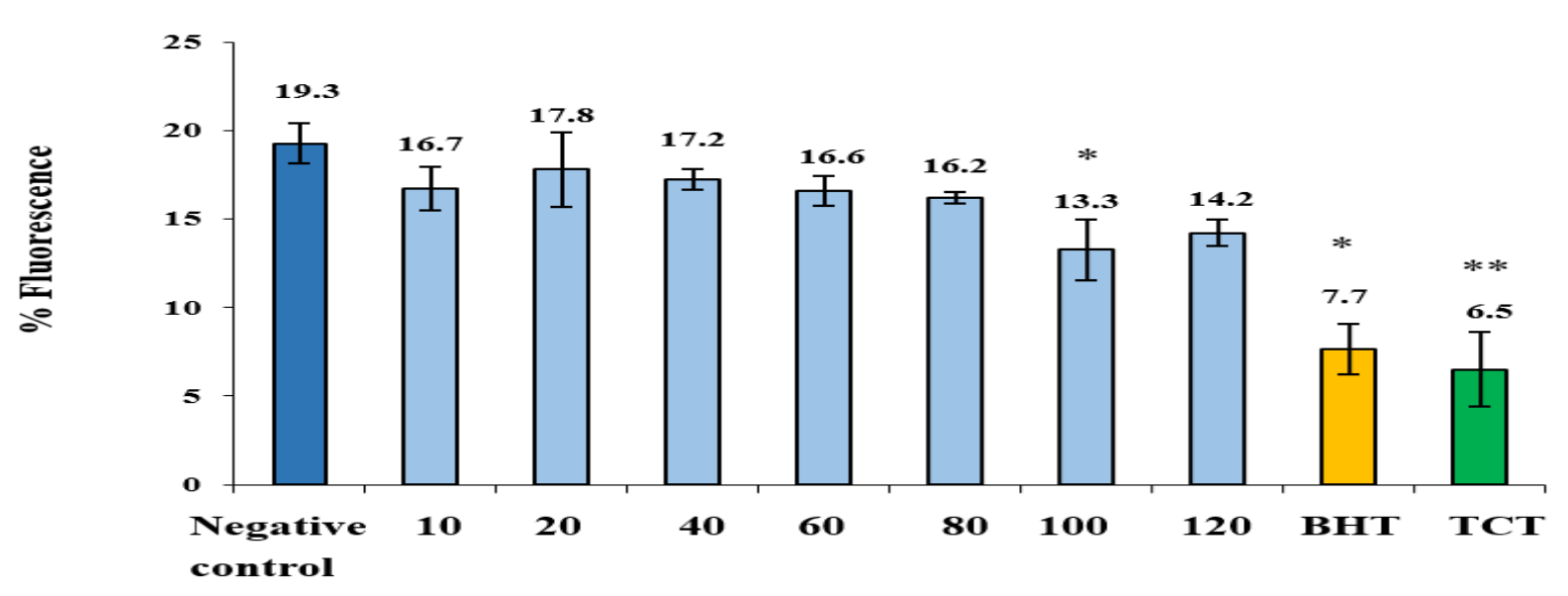

Fig.8. Percentage increase of DCF fluorescence intensity after co-incubation of various concentrations of HDL (10-120 mg/dl) with stimulated RAW 264.7 cells. BHT and TCT were used as positive controls. Each set of experiment was performed in triplicates. Results are presented as mean $\pm \mathrm{SD}$ compared to negative control, ${ }^{*} \mathrm{p}<0.05,{ }^{* *} \mathrm{p}<0.01$ (HDL: High-density lipoprotein; BHT: Butylatedhydroxytoluene; TCT: Tocotrienol)

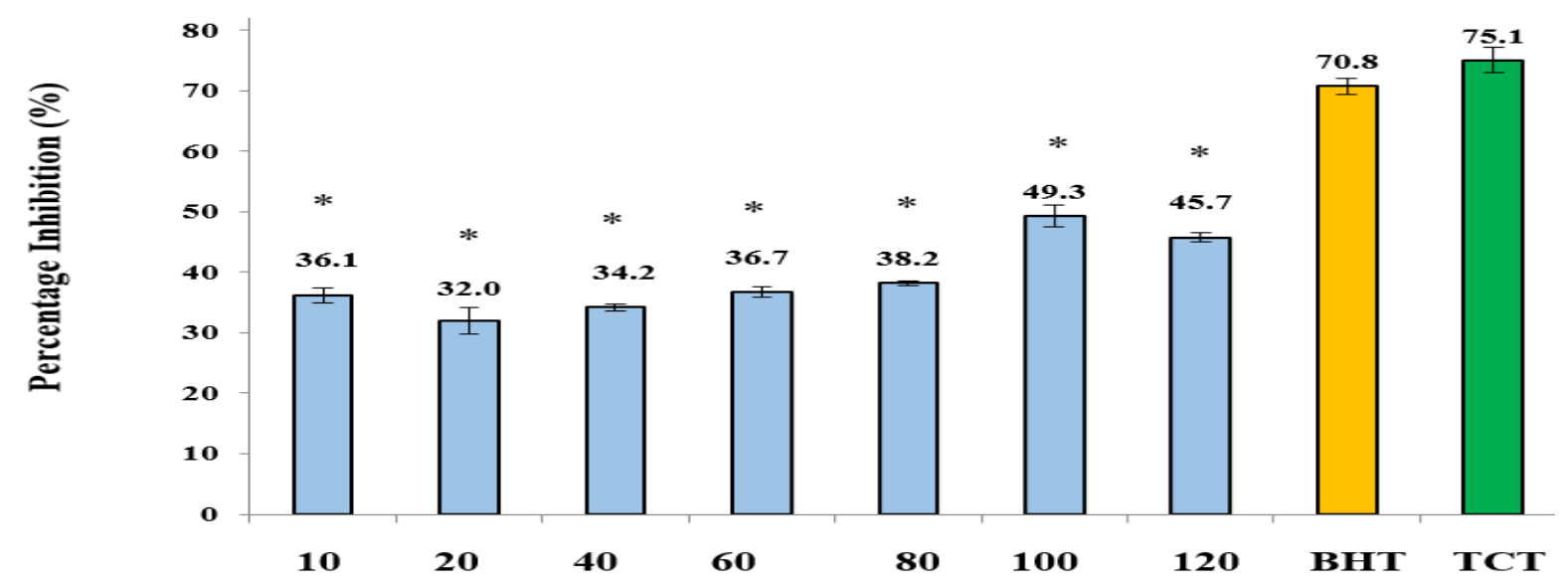

Fig.9. Percentage inhibition of ROS generation through various concentrations of HDL (10-120 mg/dl) in stimulated RAW 264.7 cells. BHT and TCT were used as positive controls.

Results are presented as mean $\pm \mathrm{SD}$ compared to positive controls, ${ }^{*} \mathrm{p}<0.05$ (BHT:

Butylatedhydroxytoluene; TCT: Tocotrienol)

The oxidation of LDL is appeared as typical conjugated diene versus time curves, where the distinctive phases of $\mathrm{H}_{2} \mathrm{O}_{2}$ formation were plainly perceivable. Coincubations of LDL with HDL created a more gradual rate of oxidation. Coincubation of LDL with HDL increased the mean lag time of diene formation across all HDL concentration in a dose-dependent manner. Incubation of LDL alone $(50 \mu \mathrm{g} / \mathrm{ml})$ as negative control showed a lag time of $35.9 \pm 5.4 \mathrm{~min}$. Coincubation of LDL with different concentrations of HDL (20, 40, 60 and $80 \mu \mathrm{g} / \mathrm{ml})$ resulted 
in longer lag time $(42.0 \pm 2.3,50.4 \pm 12.1,63.2 \pm 6.0,76.4 \pm 4.0 \mathrm{~min}, \mathrm{p}<0.05$ respectively). The amounts of lipid peroxides generated during oxidation were reduced by HDL in a concentration-dependent manner. We found that incubation with HDL in different concentrations $(20,40,60$ and $80 \mu \mathrm{g} / \mathrm{ml})$ slowed down the oxidation rate shown in Table 1 in comparison with that of incubation of LDL alone $(0.00264 \pm 0.00018,0.00241 \pm 0.0009$, $0.00145 \pm 0.0005,0.00112 \pm 0.00004$ versus $0.00379 \pm 0.00003 \mathrm{Abs} /$ minrespectively,p $<0.05$ ). Lag time or proliferation rate are indices of oxidation resistance, demonstrating the part of HDL as an antioxidant agent.

Table 1. Oxidation of $\mathrm{LDL}_{\text {by }} \mathrm{CuSO}_{4}$ within the sight of various concentrations of HDL

\begin{tabular}{lccc}
\hline & Lag Time (min) & Reaction Rate & Maximum Abs (Abs/min) \\
\hline LDL alone $(50 \mu \mathrm{g} / \mathrm{ml})$ & $35.9 \pm 5.4$ & $0.00379 \pm 0.00003$ & $0.7054 \pm 0.005$ \\
$\mathrm{LDL}+20 \mu \mathrm{g} / \mathrm{ml} \mathrm{HDL}$ & ${ }^{*} 42.0 \pm 2.3$ & ${ }^{*} 0.00264 \pm 0.00018$ & ${ }^{@} 0.6859 \pm 0.006$ \\
$\mathrm{LDL}+40 \mu \mathrm{g} / \mathrm{ml} \mathrm{HDL}$ & ${ }^{*} 50.4 \pm 12.1$ & $0.00241 \pm 0.00$ & ${ }^{\circledR} 0.7984 \pm 0.058$ \\
$\mathrm{LDL}+60 \mu \mathrm{g} / \mathrm{ml} \mathrm{HDL}$ & ${ }^{*} 63.2 \pm 6.0$ & ${ }^{*} 0.00145 \pm 0.0005$ & ${ }^{@} 0.7875 \pm 0.035$ \\
$\mathrm{LDL}+80 \mu \mathrm{g} / \mathrm{ml} \mathrm{HDL}$ & ${ }^{*} 76.4 \pm 4.0$ & ${ }^{*} 0.00112 \pm 0.00004$ & ${ }^{\circledR} 0.7788 \pm 0.008$ \\
\hline
\end{tabular}

${ }^{*} \mathrm{p}<0.05$ compared to LDL alone (Lag time), ${ }^{*} \mathrm{p}<0.05$ compared to LDL alone (reaction rate), ${ }^{\circledR} \mathrm{p}<0.05$ compared to LDL alone (Maximun absorbance).

The antioxidant properties of HDL are of great interest in view of the importance role of oxidative stress in atherogenesis[11-12]. Regardless of the different reports connecting a number of the propitious properties of HDL in the obviation of coronary heart disease thanks to its antioxidant properties, no comprehensive studies have been conducted utilizing distinctive in vitro model tests. In addition, oxidative stress is associated with endothelial dysfunction and inflammation in atherogenesis [13-14]. In atherosclerosis, oxidized LDL accumulates in the vascular wall where it is cytotoxic and chemotactic for monocytes, leading to the formation of free oxygen radicals that cause lipid peroxidation and can inactivate endothelium derived nitric oxide. The oxidation hypothesis states that the basis of atherogenesis is resulting from theoxidized LDL in the artery wall. It harms the endothelium, attracts monocytes and promotes foam cell formation. It is therefore predicted that antioxidants will be therapeutically beneficial in treating human atherosclerotic vascular disease [15]. The aim of this study was to determine the antioxidant potencies of HDL, which 
improve the antioxidant activities and to recognize the fundamental mechanisms of the antioxidant properties.

In the FTC test, the capacity of HDL to hinder lipid peroxidation is evaluated. HDL at $80 \mathrm{mg} / \mathrm{dl}$ showed highest percentage inhibition of $71.0 \pm 1.2 \%$. HDL may specifically protects LDL against oxidative modification and could secure endothelial cells against the cytotoxic impacts of LDL and that the degree of LDL lipid peroxidation was less when HDL was available. The most likely antioxidant property of HDL is thought to be on the elimination of lipid hydroperoxides (LOOH) derived from ox-LDL. Among LOOH species, nonesterified fatty acid hydroperoxides (FFA-OOH) was preferentially reduced. Two-electron reduction of the hydroperoxy group to the hydroxy group was confirmed by the generation of 13-hydroxyoctadecadienoic acid from 13-hydroperoxyoctadecadienoic acid in HPLC analyses [16]. This reducing impact was likewise found in apolipoprotein A1 (apoA1). FFA-OOH discharged from phosphatidylcholinehydroperoxides (PtdCho-OOH) due to platelet-activating factor-acetylhydrolase (PAF-AH) activity in oxidized LDL experienced two-electron diminishment by the reducing ability of apoA1 in HDL. This special reduction of FFA-OOH may involve in the mechanism of the antioxidant property of HDL [16].

Free radical-induced lipid peroxidation has been implicated in the pathogenesis of atherosclerosis, and ROS are known to be the initiators of lipid peroxidation. It is well established that critical features of atherosclerosis development are caused by the formation of ROS because ROS promote cell proliferation, hypertrophy, growth arrest, apoptosis and oxidation of LDL [17]. In the DCFHDA assay in this study, coincubation with HDL showed no effect on the cell viability regardless of HDL concentrations. HDL at $100 \mathrm{mg} / \mathrm{dl}$ has been shown to inhibit the production of $\mathrm{H}_{2} \mathrm{O}_{2}$ with a percentage inhibition of $49.3 \pm 1.7 \%$. These results clearly indicate that HDL functions as an antioxidant and inhibits ROS production, hence they may have beneficial potentials in protection against atherosclerosis. The major proteins of HDL, apoAI and apoAII and additional different proteins, for example, paraoxonase that cotransport with HDL in plasma are outstanding to have antioxidant properties [18]. There were certain limitations in the present study; the HDL isolated may still carry albumin, lipids and fat soluble vitamins which may contribute the effects observed as the HDL particles were not delipidated. In future studies, it is advisable to use a different 
isolation method that is sufficient to obtain a pure HDL fraction.

We found that HDL did not exhibit free-radical scavenging activities through the DPPH assay. It was likewise demonstrated that radical-scavenging activity of HDL diminished as the HDL concentration increased. When low concentration of HDL was added to DPPH solution free radicals were scavenged. However, when the concentration of HDL was increased the absorbance also increased. This result indicates that DPPH was not scavenged by HDL at higher concentration through donation of hydrogen to form reduced DPPH, hence suggesting that HDL has poor radical scavenging capacity. As the curve goes below zero value, it is suggested that HDL was oxidized and become a pro-oxidant. This would be in line with new evidence showed that oxidized HDL is pro-atherogenic [19]. Again, it is important to obtain higher purity of HDL than the quantity. In [20] have suggested that biologically dynamic lipids in LDL are composed in a progression of three stages. The initial step is seeding of LDL with products of linoleic acid, arachidonic acid and hydroperoxides metabolism, trailed by catching of LDL in the subendothelial space and accumulation of extra reactive oxygen species derived from the artery wall cells in LDL and the last one is the nonenzymatic oxidation of LDL phospholipids. This happens when a specific limit of ROS is reached, resulting in the development of particular oxidised phospholipids that initiate monocytes binding, chemotaxis and differentiation into macrophages. HDL will hinder each of the three stages in the formation of ox-LDL.

The initial phase of quick oxidation in such a profile, observed with LDL has been translated to happen through HDL acting as an antioxidant. From our study, it was clearly shown that HDL lengthened the lag time and slowed down LDL oxidation rate in a dose-dependent manner. This occurred because HDL bound to part of the copper. Kinetic examinations by various investigators on the impacts of HDL on copper-induced peroxidation of LDL are hard to compare due to the various concentrations of HDL, LDL, copper and the ratio of copper to lipoprotein used in this studies. In our experiment, HDL has been shown to function as a protective agent on LDL susceptibility to oxidation. Increasing the HDL concentrations lead to reduced susceptibility to LDL oxidation. The oxidized LDL is engulfed by macrophages via the scavenger receptors resulting in foam cell formation. Formation of foam cells which are highly cytotoxic to the cells of the arterial wall stimulates inflammatory and thrombotic 
processes. Macrophage-mediated oxidation of LDL is probably a key event during early atherogenesis. As HDL has antioxidant properties, it is able to inhibit oxidation. This is perhaps facilitated by the presence of a few enzymes, for example, the paraoxonase-1 (PON-1), PAF-AH and glutathione peroxidase (GPx) that are involved in atherogenesis via an inhibitory effect on lipoprotein oxidation [20-21]. Studies by [22] reported that another HDL-related enzyme lecithin:cholesterolacyltransferase (LCAT) additionally obviates the accumulation of oxidized lipids in LDL. Therefore, with elevated serum level of HDL is prevented, hence reducing the risk of LDL oxidation on atherogenesis and coronary heart disease (CHD). The results of this present study are likewise parallel with other in vitro studies which plainly demonstrated that HDL inhibits the oxidation of LDL $[23,16]$. The in vitro results of our study are in agreement with previous in vivo, human and epidemiological studies [24].

\section{CONCLUSION}

In conclusion, the present data on the antioxidant properties of HDL indicate that HDL, especially at high concentrations is an effective antioxidant through its ability to inhibit lipid peroxidation and slow down the oxidation process. HDL has been shown to have weaker inhibitory effects on ROS production and is not an active free radical scavenger. HDL reduces LDL susceptibility to oxidation in a dose-dependent manner. These recommend that HDL may exhibit beneficial effects on atherosclerosis.

\section{ACKNOWLEDGEMENTS}

This study was supported by the SAGA (Scientific Advancement Grant Allocation) research grant from the Academy of Sciences Malaysia (Grant code: M1/003).

\section{REFERENCES}

[1] Simons L A, Gibson J C. Plasma lipids and lipoproteins. In M. A. Crook (Ed.), Clinical biochemistry and metabolic medicine. Lancaster: MTP Press Limited, 2012, http://cw.taylorandfrancis.com/resources/9781444144147/sample-material/Chapter-13-Plasma -lipids-and-lipoproteins.pdf 
[2] Bastien M, Poirier P, Lemieux I, Després J P. Overview of epidemiology and contribution of obesity to cardiovascular disease. Progress in Cardiovascular Diseases, 2014, $56(4): 369-381$

[3] Rader D J, Hovingh G K. HDL and cardiovascular disease. Lancet, 2014, 384(9943):618-625

[4] Pérez-Méndez Ó, Pacheco H G, Martínez-Sánchez C, Franco M. HDL-cholesterol in coronary artery disease risk: Function or structure?ClinicaChimicaActa, 2014, 429:111-122

[5] Murphy A J, Funt S, Gorman D, Tall A R, Wang N. Pegylation of high-density lipoprotein decreases plasma clearance and enhances antiatherogenic activity novelty and significance. Circulation Research, 2013, 113(1):1-19

[6] Mooradian A D. Is high-density lipoprotein cardioprotective or simply a marker of cardiovascular disease?American Journal of Therapeutics, 2014, 21(5):438-439

[7] Umemoto T, Han C Y, Mitra P, Averill M M, Tang C, Goodspeed L, Omer M, Subramanian S, Wang S, Den Hartigh L J, Wei H, Kim E J, Kim J, O’Brien K D, Chait A. Apolipoprotein AI and high-density lipoprotein have anti-inflammatory effects on adipocytes via cholesterol transporters novelty and significance. Circulation Research, 2013, 112(10):1345-1354

[8] Mineo C, Shaul P W. Novel biological functions of high-density lipoprotein cholesterol. Circulation Research, 2012, 111(8):1079-1090

[9] Westerterp M, Wang N, Tall A R. High-density lipoproteins, endothelial function, and mendelian randomization. Circulation Research, 2016, 119(1):13-15

[10]Badimon L, Vilahur G. LDL-cholesterol versus HDL-cholesterol in the atherosclerotic plaque: inflammatory resolution versus thrombotic chaos. Annals of the New York Academy of Sciences, 2012, 1254(1):18-32, a) Lanez T. et al. Antioxidant activity and superoxide anion radical interaction with 2-(ferrocenylmethylamino) benzonitrile and 3-(ferrocenylmethylamino) benzonitrile J. Iran. Chem. Soc., 2016, 13 (9), 1741-1748, b) Lanez T. et al. Evaluation of Antioxidant Capacity of Propolis Collected in Various Areas of Algeria Using Electrochemical Techniques, Int. J. Electrochem. Sci., 2015, (10) 9641 - 9651

[11] Bandeali S, Farmer J. High-density lipoprotein and atherosclerosis: The role of antioxidant activity. Current Atherosclerosis Reports, 2012, 14(2):101-107

[12] Elsøe S, Ahnström J, Christoffersen C, Hoofnagle A N, Plomgaard P, Heinecke J W, Binder C J, Björkbacka H, Dahlbäck B, Nielsen L B. Apolipoprotein M binds oxidized 
phospholipids and increases the antioxidant effect of HDL. Atherosclerosis, 2012, 221(1):91-97

[13] Peluso I, Morabito G, Urban L, Ioannone F, Serafi M. Oxidative stress in atherosclerosis development: The central role of LDL and oxidative burst.Endocrine, Metabolic and Immune Disorders-Drug Targets (Formerly Current Drug Targets-Immune, Endocrine and Metabolic Disorders), 2012, 12(4):351-360

[14] Siti H N, Kamisah Y, Kamsiah J. The role of oxidative stress, antioxidants and vascular inflammation in cardiovascular disease (a review). Vascular Pharmacology, 2015, 71:40-56

[15] Dahech I, Harrabi B, Hamden K, Feki A, Mejdoub H, Belghith H, Belghith K S. Antioxidant effect of nondigestiblelevan and its impact on cardiovascular disease and atherosclerosis. International Journal of Biological Macromolecules, 2013, 58:281-286

[16] Kotosai M, Shimada S, Kanda M, Matsuda N, Sekido K, Shimizu Y, Tokumura A, Nakamura T, Murota K Y, Terao J. Plasma HDL reduces nonesterified fatty acid hydroperoxides originating from oxidized LDL: Amechanism for its antioxidant ability. Lipids, 2013, 48(6):569-578

[17] Rani V, Deep G, Singh R K, Palle K, Yadav U C S. Oxidative stress and metabolic disorders: Pathogenesis and therapeutic strategies. Life Sciences, 2016, 148:183-193

[18] Barter P J, Nicholls S, Rye K A, Anantharamaiah G M, Navab M, Fogelman A M. Antiinflammatory properties of HDL. Circulation Research, 2004, 95(8):764-772

[19] Soumyarani V S, Jayakumari N. Oxidatively modified high density lipoprotein promotes inflammatory response in human monocytes-macrophages by enhanced production of ROS, TNF- $\alpha$, MMP-9, and MMP-2. Molecular and Cellular Biochemistry, 2012, 366(1-2):277-285 [20] Navab M, Berliner J A, Watson A D, Hama S Y, Territo M C, Lusis A J, Shih D M, Van Lenten B J, Frank J S, Demer L L, Edwards P A, Fogelman A M. The Yin and Yang of oxidation in the development of the fatty streak. Arteriosclerosis, Thrombosis, and Vascular Biology, 1996, 16(7):831-842

[21] Berrougui H, Momo C N, Khalil A. Health benefits of high-density lipoproteins in preventing cardiovascular diseases. Journal of Clinical Lipidology, 2012, 6(6):524-533

[22] Hine D, Mackness B, Mackness M. Coincubation of PON1, APO A1, and LCAT increases the time HDL is able to prevent LDL oxidation. IUBMB Life, 2012, 64(2):157-161 
[23]Suc I, Escargueil-Blanc I, Troly M, Salvayre R, Nègre-Salvayre A. HDL and ApoAprevent cell death of endothelial cells induced by oxidized LDL. Arteriosclerosis, Thrombosis, and Vascular Biology, 1997, 17(10):2158-2166

[24] García-Heredia A, Marsillach J, Rull A, Triguero I, Fort I, Mackness B, Mackness M, Shih D M, Joven J, Camps J, Camps J. Paraoxonase-1 inhibits oxidized low-density lipoprotein-induced metabolic alterations and apoptosis in endothelial cells: Anondirectedmetabolomic study. Mediators of Inflammation, 2013, 2013:1-9

\section{How to cite this article:}

Yusoff W N W, Bakar N A A, Muid S, Ali A M, Froemming G R A, Nawawi H. Antioxidant activity of high-density lipoprotein (hdl) using different in vitro assays. J. Fundam. Appl. Sci., 2017, 9(6S), 298-315. 